\title{
Association between BHMT gene rs3733890 polymorphism and cancer risk: evidence from a meta-analysis
}

This article was published in the following Dove Press journal:

OncoTargets and Therapy

22 August 2016

Number of times this article has been viewed

Yue $\mathrm{Xu}{ }^{1, *}$

Cunye Yan ${ }^{2, *}$

Zongyao $\mathrm{Hao}^{\prime}$

Jun Zhou'

Song Fan'

Sheng Tai'

Cheng Yang'

Li Zhang'

Chaozhao Liang'

'Department of Urology, The First Affiliated Hospital of Anhui Medical University and Institute of Urology,

${ }^{2}$ First School of Clinical Medicine, Anhui Medical University, Hefei, Anhui, People's Republic of China

*These authors contributed equally to this work
Background and objective: The gene betaine-homocysteine methyltransferase (BHMT) has drawn much attention during the past decades. An increasing number of clinical and genetic investigations have supposed that BHMT rs3733890 polymorphism might be associated with risk of breast cancer and ovarian cancer. As no consistent conclusion has been achieved, we conducted an up-to-date summary of BHMT rs3733890 polymorphism and cancer risk through a meta-analysis.

Materials and methods: The articles were collected from PubMed, Google Scholar, and CNKI (Chinese) databases up to December 2015. Then, the correlations were determined by reading the titles and abstracts and by further reading the full text to filter the unqualified articles. Odds ratio (OR) and the corresponding 95\% confidence intervals (CI) were used to assess the results.

Results: Among 187 articles collected in the analysis, seven studies with a total of 2,832 cases and 3,958 controls were included for evaluation of the association between BHMT rs3733890 polymorphism and susceptibility of cancer risk. The heterogeneity test showed no significant differences. Furthermore, we found that $B H M T-742 \mathrm{G}>$ A polymorphism in case and control groups showed no statistically significant association with susceptibility in various cancer types except for uterine cervical cancer (A vs G: OR $=0.641,95 \% \mathrm{CI}=0.445-0.923, P=0.017$; $\mathrm{AA}+\mathrm{AG}$ vs $\mathrm{GG}: \mathrm{OR}=0.579,95 \% \mathrm{CI}=0.362-0.924, P=0.022$ ). In addition, no statistically significant association was uncovered when stratification analyses were conducted by ethnicity and genotyping methods.

Conclusion: Our results have shown no obvious evidence that rs3733890 polymorphism in BHMT gene affected the susceptibility of head and neck squamous cell carcinoma, breast cancer, ovarian cancer, colorectal adenoma, and liver cancer. In contrast, we found the protective role of $B H M T-742 \mathrm{G}>\mathrm{A}$ polymorphism in uterine cervical cancer incidence. Future well-designed studies comprising larger sample size are warranted to verify our findings.

Keywords: BHMT, polymorphism, cancer risk, susceptibility, meta-analysis

\section{Introduction}

Malignant tumors are still one of the leading causes of death on a global scale. According to the latest statistics, in 2015, about 589,430 Americans are estimated to die of cancer, or about 1,620 people per day. Cancer is the second most common cause of death in the US, is exceeded only by heart disease, and accounts for nearly one of every four deaths. ${ }^{1}$ Moreover, the death rate of cancer continuously increases due to the lack of early cancer detection such as widespread screening of cancer biomarkers. 
Genetic polymorphisms have been widely accepted to play a significant role in human diseases. In recent years, the relationship between genetic polymorphisms and the risk of cancers has been extensively investigated. A large number of recent studies have shown that DNA utility could be regarded as a cancer-related biomarker, which is supported by the finding that some DNA displayed altered expression profiles in cancers compared with matched normal tissues. A large amount of genes, including betaine-homocysteine methyltransferase (BHMT), have been confirmed to contribute to the complex molecular mechanisms involved in the control of cell differentiation, growth, and survival processes, which are tightly related to cancer development and progression.

The human BHMT gene has been mapped to chromosome 5q13.1-q15, ${ }^{2}$ and a common single nucleotide polymorphism (c.742G $>$ A; rs3733890), which replaces an arginine by a glutamine at codon 239 (R239Q). ${ }^{3}$ Human BHMT gene is supposed to produce an enzyme with higher affinity to homocysteine than the wild type. ${ }^{4}$ This polymorphism possibly plays a critical role in Hcy homeostasis. We have found the approximated frequency of 0.30 for BHMT $742 \mathrm{G}>\mathrm{A}$ according to the studies. ${ }^{5-10}$ Concretely, the allelic frequencies described in control samples were $0.25-0.33$ in the US, ${ }^{5} 0.31$ in Canada, ${ }^{6} 0.28$ and 0.29 in Poland, ${ }^{7} 0.30$ in Romania, ${ }^{8} 0.30$ in People's Republic of China, ${ }^{9}$ and 0.31 in the Netherlands. ${ }^{10}$

In 2007, Hazra et $\mathrm{al}^{11}$ suggested that the association between BHMT polymorphism and cancer for the first time in a study about 24 related gene polymorphisms related to colorectal cancer in the one-carbon metabolic pathway. A subsequent study ${ }^{6}$ has mentioned BHMT gene polymorphisms and tumor susceptibility. Moreover, a recent study ${ }^{12}$ that aimed to explore the molecular mechanisms involved in the association between abnormal transcription of BHMT and liver cancer risk has indicated a significant reduction in $B H M T$ gene expression in HepG2 cells and matched cancerous/adjacent normal liver samples from patients, which provided the explanation for the decreased BHMT mRNA levels previously reported in tumor tissue ${ }^{13}$ and the decreased BHMT protein in hepatocellular carcinoma. ${ }^{14,15}$ Therefore, we can infer that it has a close relationship between polymorphisms of BHMT and other cancer susceptibility, including uterine cervical cancer, ${ }^{16}$ ovarian cancer, ${ }^{17}$ and colorectal adenoma. ${ }^{11}$ These cancer types were taken as candidates to know the associations between BHMT polymorphisms and cancer susceptibility. Current individual studies did not have enough efficiency to elaborate their association. Therefore, we conducted the present meta-analysis to derive a more precise result of the relationship between BHMT rs3733890 polymorphism and cancer risk by pooling all available data together.

\section{Methods}

\section{Literature search strategy}

The articles were collected from PubMed, Google Scholar, and CNKI (Chinese). The keywords were (BHMT OR betaine homocysteine methyltransferase) AND (polymorphism OR SNP OR variant OR mutation) AND (cancer OR tumor OR carcinoma OR neoplasm OR malignancy). Meanwhile, we selected the studies that have been published in Chinese or English by December 2015 to determine the correlation by reading titles and abstracts, and read the full text to filter the unqualified articles.

\section{Identification of eligible studies}

We enrolled the studies that met the following criteria: 1) the inclusion of the literature is a case-control study; 2) the data can be extracted from the case group and the control group; 3) the studies provide plenitudinous data for calculating the odds ratio (OR) and the corresponding 95\% confidence intervals $(95 \% \mathrm{CI})$; and 4$)$ detailed genotyping data were recorded in the study.

\section{Quality score evaluation}

Data were disposed independently by two authors (Y Xu and C Yan). A consensus was finally reached by comprehensively comparing the data, and extensive discussion. Then, the following information from each included study was extracted: first author, publication year, ethnicity, genotyping method, source of control groups, cancer types, and the number of cases and controls.

\section{Statistical analysis and publication bias evaluation}

STATA 12.0 software version (STATA Corp, College Station, TX, USA) was used for statistical analysis. The associations with cancer risk were detected underlying genotyping models, including allele comparision, recessive model, dominant model, homozygote model and heterozygote model. Computation corresponding to OR and $95 \%$ CI of the selected case-control studies was employed to evaluate the association between the BHMT polymorphism and cancer risk. The publication bias was evaluated by the Egger regression and Begg's funnel plots test. $P<0.05$ means statistically significant. $P^{\mathrm{a}}$ means $P$-value of $Q$-test for heterogeneity test. The index is used to evaluate the heterogeneity. 


\section{Results}

\section{Description of search results}

As shown in Figure 1, 24 studies were retrieved initially using the search strategy described in the Methods section. After reading the title or abstract, we excluded 13 irrelevant studies. We further evaluated the remaining eleven potential relevant studies by reading the full-length text. Four studies were excluded due to lack of detailed genotyping data.

Finally, seven articles (including study stages) were selected for meta-analysis. The main characteristics of the seven study stages for the meta-analysis are shown in Table 1. For BHMT rs3733890 polymorphisms, 2,832 cases and 3,958 controls were enrolled in our analysis, the ethnicities consisted of Asian (one study), Caucasian (five studies), and mix (one study). Among the genotyping methods of these studies, three were polymerase chain reactionrestriction fragment length polymorphism, and the others were TaqMan. The sources of control were from hospital, and the types of cancer included head and neck squamous cell carcinoma, breast cancer, uterine cervical carcinoma, and ovarian cancer.

\section{Meta-analysis}

The results of meta-analysis for rs3733890 polymorphism in BMHT and cancer susceptibility are shown in Table 2. According to the results of analysis, we found that the distribution of G742A genotype showed no statistically significant differences in the case and control groups. In the subgroup analyses, performed by ethnicity and genotyping methods, we revealed a negative result (Table 2).

Meanwhile, from the forest plots, significantly decreased associations were observed in uterine cervical carcinoma regarding $B H M T-742 \mathrm{G}>\mathrm{A}$ polymorphism (A vs $\mathrm{G}$ : $\mathrm{OR}=0.641,95 \% \mathrm{CI}=0.445-0.923, P=0.017 ; \mathrm{AA}+\mathrm{AG}$ vs $\mathrm{GG}$ : $\mathrm{OR}=0.579,95 \% \mathrm{CI}=0.362-0.924, P=0.022$ ) (Figure 2). No significant associations were detected in head and neck squamous cell carcinoma, breast cancer, ovarian cancer, colorectal adenoma, and liver cancer regarding BHMT $-742 \mathrm{G}>\mathrm{A}$ polymorphism (Table $\mathrm{S} 1$ ).

\section{Sensitivity analyses and publication bias}

Sensitivity analysis for BHMT rs3733890 polymorphism and cancer risk was conducted by removing one individual study a time from the pooled OR (Figure 3), whereas the overall statistical significance did not change, indicating that the results are stable.

Begg's funnel plot and Egger's regression were also performed to evaluate the publication bias. The Begg's funnel plot of BHMT rs3733890 polymorphism and cancer risk for allelic comparison is shown in Figure 4; it seemed symmetrical, indicating the nonexistence of publication bias. Egger's test was used to assess for publication bias. According

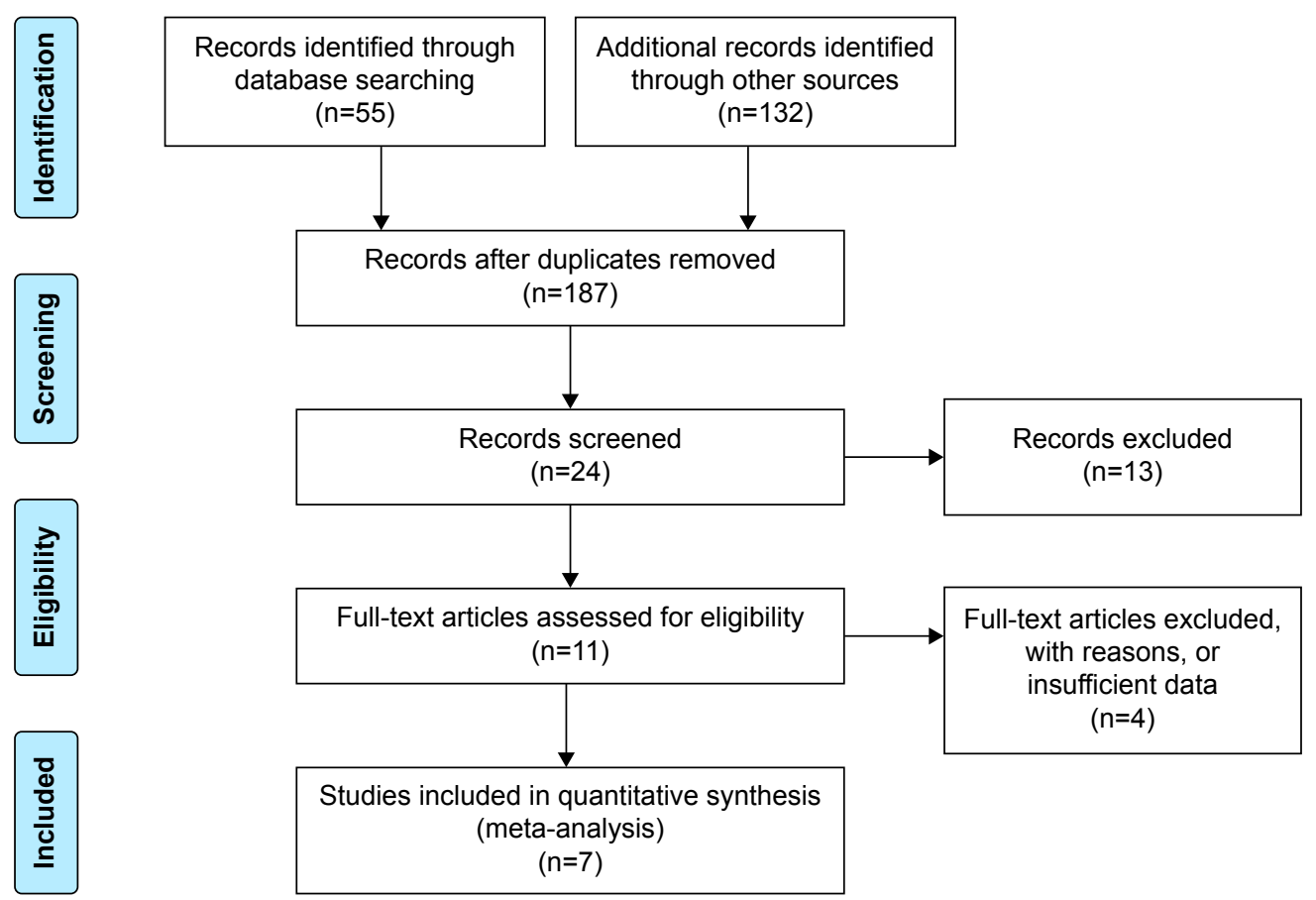

Figure I Flow diagram of the inclusion and exclusion of studies in this meta-analysis. 
Table I Main characteristics of studies regarding the association between BHMT gene rs3733890 polymorphism and cancer risk

\begin{tabular}{|c|c|c|c|c|c|c|c|c|c|c|c|c|}
\hline \multirow[t]{2}{*}{ SNP } & \multirow[t]{2}{*}{ Authors } & \multirow[t]{2}{*}{ Year } & \multirow[t]{2}{*}{ Ethnicity } & \multirow{2}{*}{$\begin{array}{l}\text { Genotyping } \\
\text { method }\end{array}$} & \multirow{2}{*}{$\begin{array}{l}\text { Source } \\
\text { of control }\end{array}$} & \multirow[t]{2}{*}{ Cancer type } & \multicolumn{3}{|c|}{ Cases } & \multicolumn{3}{|c|}{ Controls } \\
\hline & & & & & & & GG & GA & AA & GG & GA & AA \\
\hline \multirow[t]{7}{*}{ rs3733890 } & de Silva et al $^{18}$ & 2012 & Mix & PCR-RFLP & PB & HNSCC & 117 & 119 & 36 & 212 & 227 & 51 \\
\hline & $X u$ et $a^{6}$ & 2008 & Caucasian & TaqMan & $\mathrm{CB}$ & $\mathrm{BC}$ & 510 & 443 & 108 & 530 & 456 & 122 \\
\hline & Mostowska et al ${ }^{16}$ & 2011 & Caucasian & PCR-RFLP & $\mathrm{HB}$ & UCC & 70 & 46 & 8 & 72 & 77 & 19 \\
\hline & Pawlik et al ${ }^{17}$ & 2012 & Caucasian & PCR-RFLP & $\mathrm{HB}$ & OC & 64 & 47 & 23 & 67 & 76 & 17 \\
\hline & Hazra et al" & 2007 & Caucasian & TaqMan & $\mathrm{HB}$ & CRA & 40 & 237 & 248 & 57 & 223 & 245 \\
\hline & Xu et al ${ }^{19}$ & 2008 & Caucasian & TaqMan & PB & $\mathrm{BC}$ & 192 & 183 & 43 & 128 & 108 & 31 \\
\hline & $\mathrm{An}^{20}$ & 2008 & Asian & TaqMan & PB & LC & 315 & 310 & 73 & 557 & 545 & 138 \\
\hline
\end{tabular}

Abbreviations: BC, breast cancer; CB, community-based; CRA, colorectal adenoma; HB, hospital-based; HNSCC, head and neck squamous cell carcinoma; LC, liver cancer; OC, ovarian cancer; PB, population-based; PCR-RFLP, polymerase chain reaction-restriction fragment length polymorphism; SNP, single nucleotide polymorphism; UCC, uterine cervical carcinoma.

to Egger's test, we found no evidence of publication bias (A vs G, Egger's $P=0.573$, Begg's $P=0.764$ ).

\section{Quality assessment}

Generally, it is well established to assess the methodological "quality" of included studies based on the Newcastle-Ottawa scale for quality of case-control studies and cohort studies in meta-analysis. For this assessment, we used the star system (ranged from zero to nine stars) and considered a study awarded five or more stars as a high-quality study. ${ }^{21}$ The values of the seven case-control studies ranged from six stars to eight stars (Table 3 ).

\section{Discussion}

Over the past decades, the role of polymorphisms in gene encoding enzymes of BHMT metabolism has drawn much attention. BHMT has been detected in eukaryotes and prokaryotes. $^{22-24}$ Until now, most of the previous studies have been carried out in mammals, and the protein levels could be detected generally at day 10 after gestation or in adults. ${ }^{22,25}$ The $B H M T$ gene is polymorphic in the nucleotide $742 \mathrm{G}>\mathrm{A}$, with a substitution of arginine for glutamine in the protein. ${ }^{26}$ These polymorphisms are believed to contribute to the risk for liver cancer, although the mechanism by which this may occur is not clearly understood. Plenty of molecular epidemiologic studies have evaluated the role of BHMT polymorphism in different cancer. $\mathrm{Xu}$ et $\mathrm{al}^{6}$ conducted a case-control study, which enrolled 1,065 cases and 1,109 controls. The BHMT rs3733890 polymorphism has been reported previously in this population, but it was not associated with breast cancer risk. Then, in order to verify Xu et al's findings, Mostowska et al ${ }^{16}$ conducted a case-control study, which enrolled 142 cases and

Table 2 Results of meta-analysis for the association between BMHT gene polymorphism and cancer susceptibility

\begin{tabular}{|c|c|c|c|c|c|c|c|c|c|c|}
\hline \multirow{2}{*}{$\begin{array}{l}\text { Variables } \\
\text { (rs3733890) }\end{array}$} & \multirow{2}{*}{$\begin{array}{l}\text { Casel } \\
\text { control }\end{array}$} & \multicolumn{3}{|l|}{ A vs $\mathbf{G}$} & \multicolumn{3}{|l|}{ AA vs GG } & \multicolumn{3}{|l|}{ AG vs GG } \\
\hline & & OR (95\% CI) & $P^{a}$-value & $I^{2}(\%)$ & OR $(95 \% \mathrm{CI})$ & $P^{a}$-value & $I^{2}(\%)$ & OR (95\% CI) & $P^{a}$-value & $I^{2}(\%)$ \\
\hline Total & $2,832 / 3,958$ & $0.992(0.924-1.066)$ & 0.305 & 16.4 & $1.013(0.863-1.188)$ & 0.203 & 29.6 & $0.980(0.839-1.145)$ & 0.099 & 43.8 \\
\hline \multicolumn{11}{|c|}{ Genotyping method } \\
\hline PCR-RFLP & $530 / 818$ & $0.950(0.806-1.120)$ & 0.057 & 65.2 & $\mathrm{I} .077(0.752-\mathrm{I} .542)$ & 0.079 & 60.7 & $0.765(0.569-1.028)$ & 0.233 & 31.4 \\
\hline TaqMan & $2,302 / 3,140$ & $1.002(0.926-1.085)$ & 0.771 & 0.0 & $0.997(0.835-1.192)$ & 0.357 & 7.3 & I.056 (0.933-I.I95) & 0.365 & 5.6 \\
\hline \multicolumn{11}{|l|}{ Ethnicity } \\
\hline Caucasian & & $0.985(0.901-1.077)$ & 0.155 & 40.0 & I.005 (0.822-I.229) & 0.117 & 45.8 & $0.96 \mathrm{I}(0.740-\mathrm{I} .248)$ & 0.032 & 62.2 \\
\hline Asian & & $0.980(0.852-1.127)$ & & & $0.935(0.682-1.282)$ & & & $1.006(0.827-1.224)$ & & \\
\hline \multirow[t]{2}{*}{ Mix } & & I.07I (0.859-I.335) & & & I.279 (0.789-2.073) & & & $0.950(0.692-1.304)$ & & \\
\hline & & $\mathbf{A A}+\mathbf{A} \mathbf{G}$ vs $\mathbf{G} \mathbf{G}$ & & & AA vs $\mathbf{A G}+\mathbf{G G}$ & & & & & \\
\hline Total & $2,832 / 3,958$ & $0.990(0.896-1.093)$ & 0.135 & 38.5 & $0.991(0.865-1.135)$ & 0.334 & 12.6 & & & \\
\hline \multicolumn{11}{|c|}{ Genotyping method } \\
\hline PCR-RFLP & $530 / 818$ & $0.842(0.676-1.050)$ & 0.138 & 49.6 & $1.210(0.86 \mathrm{I}-\mathrm{I} .700)$ & 0.100 & 56.6 & & & \\
\hline TaqMan & $2,302 / 3,140$ & I.03I (0.923-I.I53) & 0.355 & 7.6 & $0.955(0.823-1.107)$ & 0.907 & 0.0 & & & \\
\hline \multicolumn{11}{|l|}{ Ethnicity } \\
\hline Caucasian & & $0.985(0.867-1.120)$ & 0.045 & 58.9 & $0.974(0.829-1.145)$ & 0.267 & 23.1 & & & \\
\hline Asian & & $0.992(0.823-1.195)$ & & & $0.933(0.691-1.259)$ & & & & & \\
\hline Mix & & $1.010(0.749-1.363)$ & & & $1.313(0.833-2.070)$ & & & & & \\
\hline
\end{tabular}

Notes: $P^{2}$ (\%): 0-25, no heterogeneity; $25-50$, modest heterogeneity; $>50$, high heterogeneity; a $P$-value of $Q$-test for heterogeneity test.

Abbreviations: $\mathrm{Cl}$, confidence interval; OR, odds ratio; PCR-RFLP, polymerase chain reaction-restriction fragment length polymorphism. 
A

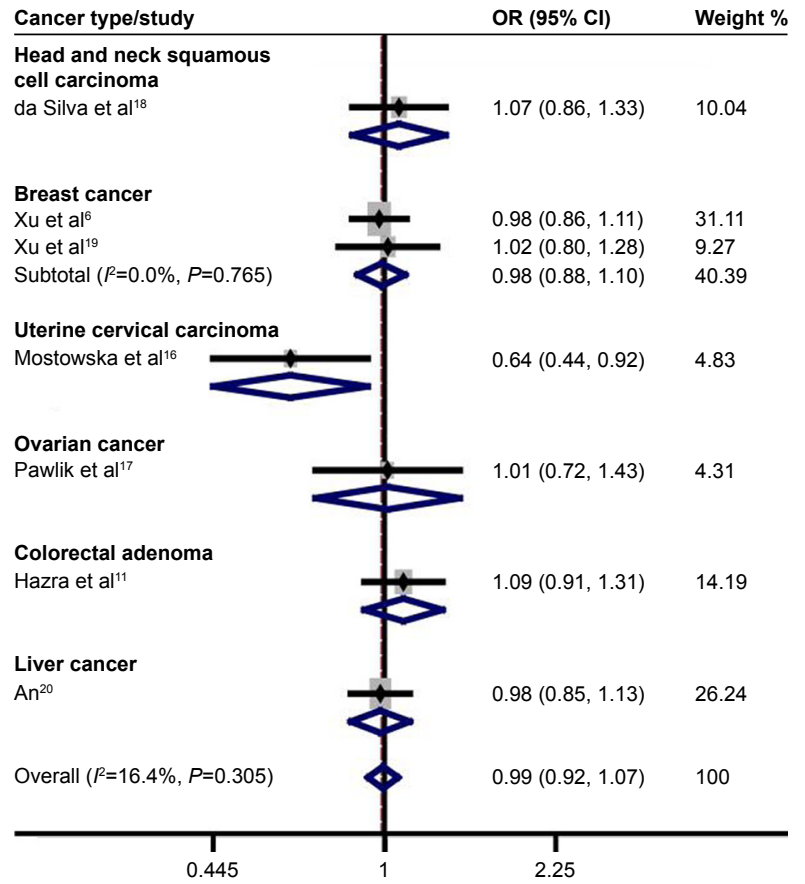

B

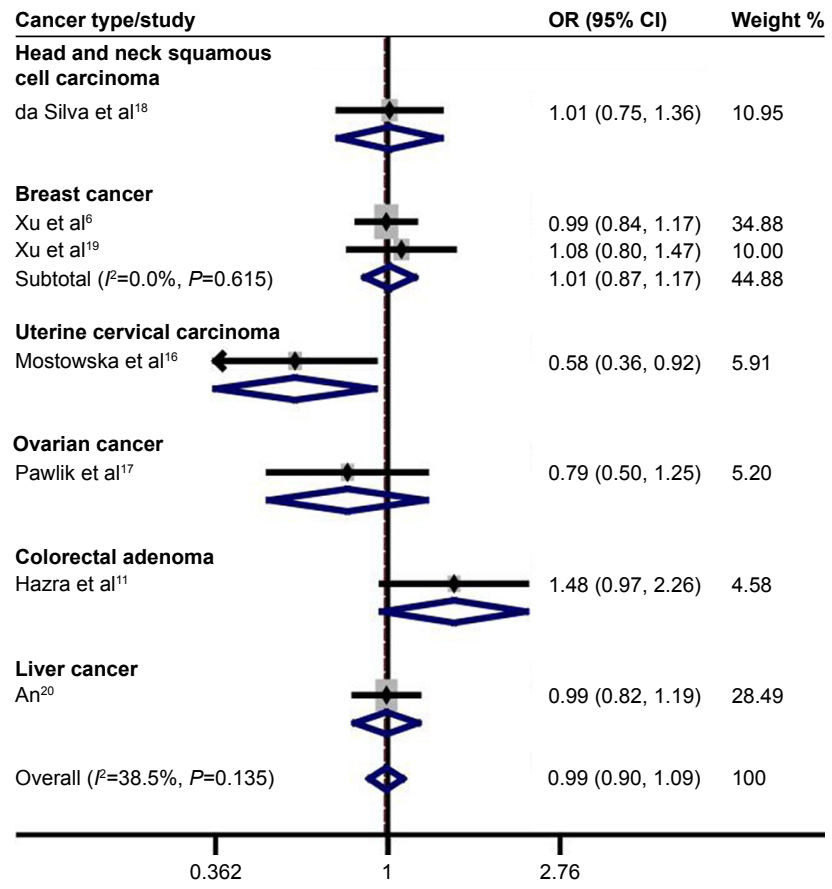

Figure 2 Forest plots describing the meta-analysis for the association between the BHMT rs373389 polymorphism and cancer risk.

Notes: (A) Allele contrast (A vs G) and (B) dominant model (AA+AG vs GG). Each square indicates a study, and the area of the squares is proportional to the weight of the study. The diamond represents the summary OR, and the transverse line means $95 \% \mathrm{Cl}$.

Abbreviations: $\mathrm{Cl}$, confidence interval; $\mathrm{OR}$, odds ratio.

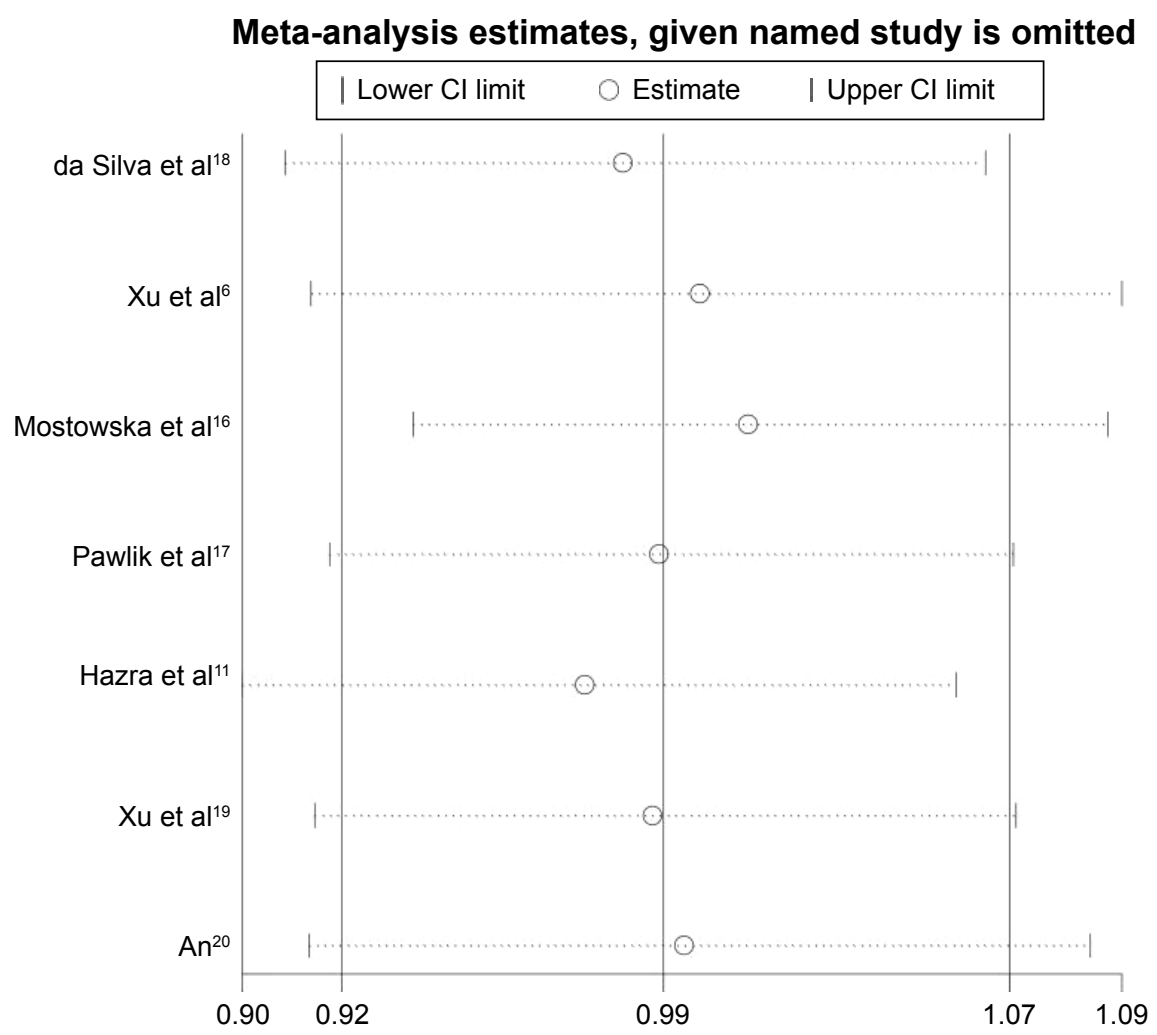

Figure 3 Sensitivity analysis of BHMT rs 3733890 polymorphism in allelic comparison (A vs G).

Notes: The middle vertical solid line is the estimated line. The left-most line is the lower Cl limit. The right-most line is the upper $\mathrm{Cl}$ limit. Each circle is a separate study and indicates OR. The dotted line means $95 \% \mathrm{Cl}$.

Abbreviations: $\mathrm{Cl}$, confidence interval; $\mathrm{OR}$, odds ratio. 


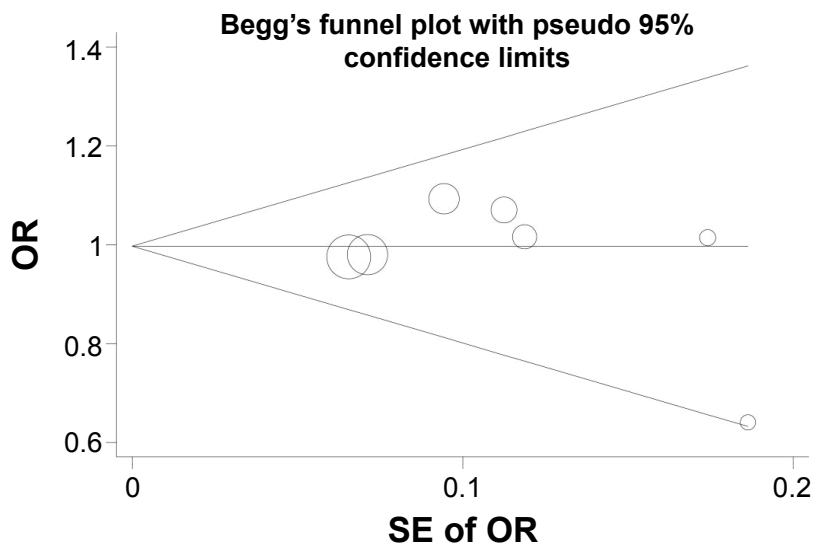

Figure 4 Begg's funnel plot for publication bias test of BHMT rs3733890 polymorphism in allelic comparison (A vs $G$ ).

Notes: The $x$-axis is $\log (O R)$ and the $y$-axis is natural logarithm of OR. The horizontal line in the figure means the overall estimated log (OR). The two diagonal lines indicate the pseudo $95 \%$ confidence limits of the effect estimate.

Abbreviations: OR, odds ratio; log (OR), log-transformed OR; SE, standard error.

168 controls, and they identified that GG and AG genotype of BHMT polymorphism had a 1.6- and 1.2-fold increased risk for cervical cancer. In addition, a study that included a total of 762 individuals (272 patients with head and neck cancer and 490 controls), conducted by da Silva et al, ${ }^{18}$ suggests that BHMT G742A associated to tobacco increases head and neck squamous cell carcinoma risk. As the result remains controversy in the association between BHMT rs 3733890 polymorphism and cancer risk, meta-analysis is regarded as a crucial method to accurately define the influence of specific genetic polymorphisms on cancer susceptibility. However, the association between BHMT polymorphism and other tumor susceptibility has still not been found. In the stratification analyses for BHMT rs3733890 polymorphism by ethnicity, genotyping method or control source, no significant association was observed in the subgroups.

Malignant tumor is a complex multi-gene genetic disease; several factors can cause diverse research results in revealing the possible correlations between cancer risk and gene polymorphisms. Among the influential factors, racial specificity, environmental stress, living habits, and unclear interactions between identified and unidentified genes might play important roles. Particularly, there has been accumulating evidence regarding the joint effects of commonly occurring single nucleotide polymorphisms (SNPs) on cancer risks, ${ }^{27-30}$ supported by polygenic models in various cancer types including breast, ${ }^{31}$ colorectum, ${ }^{32}$ head/neck, ${ }^{33}$ oral cavity, ${ }^{34}$ liver, ${ }^{30}$ cervical, ${ }^{35}$ and ovarian cancer. ${ }^{36}$ Most of these studies have focused on the interactions of genome-wide SNPs, which are located in different chromosomes. Consistent with our findings, most aforementioned studies have addressed that

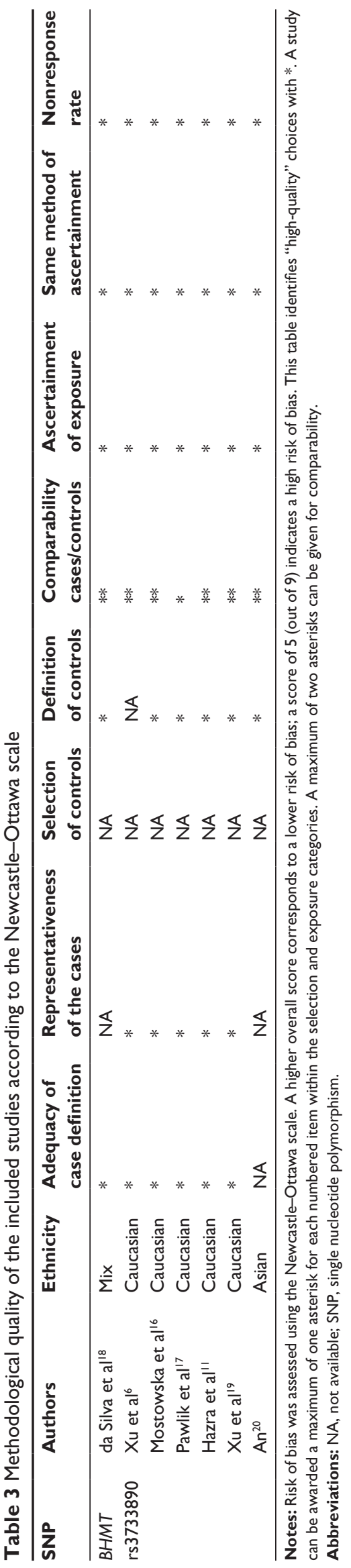


the effects of some SNPs could be categorized as "not associated" and further concluded that they were not important in cancer risks. The possible explanation was that some SNPs might not possess main effects or only possessed negligible effects to interact with other SNPs and subsequently conferred a changed risk for cancers. ${ }^{37}$ Meanwhile, the limited eligible studies may further lead to the lack of statistically significant differences.

The underlying mechanisms of the carcinogenesis are obscure because of the involvement of multiple risk factors containing complicated gene-gene and gene-environment interactions. ${ }^{38}$ Although considerable retrieval and analysis have been done, the following limitations exist. Firstly, the eligible studies were limited and the corresponding sample size was made relatively small. A large sample size and multicenter study is needed to confirm the reliability of our conclusion. Secondly, the impact of the differences in population genetic structure should not be ignored. The site itself is not a lethal site. It is in a linkage disequilibrium with the adjacent real lethal sites in some populations, whereas in other populations, there is no linkage disequilibrium, which determined that the site is associated with tumor susceptibility. Thirdly, we recognize that the possible BHMT gene SNP-SNP interaction or jointed effect of SNPs is important to comprehensively investigate their roles in various cancerous initiation and progression, which issues we should not ignore. In this meta-analysis, we have obtained only one qualified BHMT gene polymorphism rs3733890; therefore, it is impossible to evaluate the SNP-SNP interaction or jointed effect of SNPs within BHMT gene polymorphisms themselves. In contrast, a previous study ${ }^{20}$ has explored the possible joint effects among the 20 critical candidate genes (MTHFR, TS, MTR, MTRR, MTHRDI, PEMT, CHDH, BHMT, SHMTI, CHKA, SLC19AI, TCNZ, FOLRI, HCPI, GNMT, DPYD, ABCB4, DNMTI, CBS, and DHFR) involved in the one-carbon metabolism network, which is regarded as an important role on DNA synthesis. Methylation linked the genetic and epigenetic progression closely associated with the development and prevention of several malignancies, and eventually it was found that no positive or meaningful SNP-SNP interactions were associated with BHMT gene polymorphisms. However, based on the aforementioned results, we still cannot exclude the possibility that there will be novel SNPs, which could interact with BHMT rs3733890. Therefore, we will continue to focus on the progress of the related research studies and make the necessary update. Besides, other external causes, such as individual persons usually have different genetic backgrounds, the differences of the external environment and the susceptibility genes were important influential factors in the current study.

\section{Conclusion}

Our study showed that there was no statistically significant association between G742A BHMT gene polymorphism and the susceptibility of various cancer types including head and neck squamous cell carcinoma, breast cancer, ovarian cancer, colorectal adenoma, and liver cancer. In contrast, we found the protective role of $B H M T-742 \mathrm{G}>\mathrm{A}$ polymorphism in uterine cervical cancer incidence (A vs G: OR $=0.641,95 \%$ $\mathrm{CI}=0.445-0.923, P=0.017 ; \mathrm{AA}+\mathrm{AG}$ vs $\mathrm{GG}: \mathrm{OR}=0.579$, $95 \% \mathrm{CI}=0.362-0.924, P=0.022)$. In the future, well-designed studies comprising larger sample size are warranted to further verify these findings.

\section{Acknowledgments}

This meta-analysis was supported by grants from the Clinical Key Subjects Program of the Ministry of Public Health (Urology), the National Natural Science Foundation of China (81370856 and 81401518), Anhui Provincial Natural Science Foundation (1408085QH180), the cultivation project for National Natural Science Foundation of China at Anhui Medical University (2013KJ14), and the cultivation project for Young Top-Notch Talent Support Scheme from Anhui Medical University. The funders had no role in study design, data collection and analysis, decision to publish, or preparation of the manuscript.

\section{Disclosure}

The authors report no conflicts of interest in this work.

\section{References}

1. Siegel RL, Miller KD, Jemal A. Cancer statistics, 2015. CA Cancer J Clin. 2015;65(1):5-29.

2. Sunden SL, Renduchintala MS, Park EI, Miklasz SD, Garrow TA. Betaine-homocysteine methyltransferase expression in porcine and human tissues and chromosomal localization of the human gene. Arch Biochem Biophys. 1997;345(1):171-174.

3. Morin I, Platt R, Weisberg I, et al. Common variant in betaine-homocysteine methyltransferase (BHMT) and risk for spina bifida. Am JMed Genet. 2003; 119A:172-173.

4. Li F, Feng Q, Lee C, et al. Human betaine-homocysteine methyltransferase (BHMT) and BHMT2: common gene sequence variation and functional characterization. Mol Genet Metab. 2008;94(3):326-335.

5. Weisberg IS, Park E, Ballman KV, et al. Investigations of a common genetic variant in betaine-homocysteine methyltransferase (BHMT) in coronary artery disease. Atherosclerosis. 2003;167(2):205-214.

6. Xu X, Gammon MD, Zeisel SH, et al. Choline metabolism and risk of breast cancer in a population-based study. FASEB J. 2008;22(6): 2045-2052.

7. Mostowska A, Hozyasz KK, Wojcicki P, Dziegelewska M, Jagodzinski PP. Associations of folate and choline metabolism gene polymorphisms with orofacial clefts. J Med Genet. 2010;47(12):809-815. 
8. Popp RA, Farcas MF, Trifa AP, Crisan TO, Militaru MS, Pop IV. Association of betaine-homocysteine S-methyltransferase gene G742A SNP and male infertility. Rev Rom Med Labor. 2012;20(1/4):57-62.

9. Hu Y, Chen EJ, Mu Y, Li JL, Chen RJ. BHMT gene polymorphisms as risk factors for cleft lip and cleft palate in a Chinese population. Biomed Environ Sci. 2011;24(2):89-93.

10. Heil SG, Lievers KJ, Boers GH, et al. Betaine-homocysteine methyltransferase (BHMT): genomic sequencing and relevance to hyperhomocysteinemia and vascular disease in humans. Mol Genet Metab. 2000;71(3):511-519.

11. Hazra A, Wu K, Kraft P, et al. Twenty-four non-synonymous polymorphisms in the one-carbon metabolic pathway and risk of colorectal adenoma in the Nurses' Health Study. Carcinogenesis. 2007;28(7): 1510-1519.

12. Pellanda $H$, Namour $F$, Fofou-Caillierez $M$, et al. A splicing variant leads to complete loss of function of betaine-homocysteine methyltransferase (BHMT) gene in hepatocellular carcinoma. Int J Biochem Cell Biol. 2012;44(2):385-392.

13. Avila MA, Berasain C, Torres L, et al. Reduced mRNA abundance of the main enzymes involved in methionine metabolism in human liver cirrhosis and hepatocellular carcinoma. J Hepatol. 2000;33(6):907-914.

14. Liang CR, Leow CK, Neo JC, et al. Proteome analysis of human hepatocellular carcinoma tissues by two-dimensional difference gel electrophoresis and mass spectrometry. Proteomics. 2005;5(8):2258-2271.

15. Sun W, Xing B, Sun Y, et al. Proteome analysis of hepatocellular carcinoma by two-dimensional difference gel electrophoresis: novel protein markers in hepatocellular carcinoma tissues. Mol Cell Proteomics. 2007;6(10):1798-1808.

16. Mostowska A, Myka M, Lianeri M, Roszak A, Jagodziński PP. Folate and choline metabolism gene variants and development of uterine cervical carcinoma. Clin Biochem. 2011;44(8-9):596-600.

17. Pawlik P, Mostowska A, Lianeri M, et al. Folate and choline metabolism gene variants in relation to ovarian cancer risk in the Polish population. Mol Biol Rep. 2012;39(5):5553-5560.

18. da Silva LM, Galbiatti AL, Ruiz MT, et al. MTHFD1 G1958A, BHMT G742A, TC2 C776G and TC2 A67G polymorphisms and head and neck squamous cell carcinoma risk. Mol Biol Rep. 2012;39(2):887-893.

19. Xu XR, Gammon MD, Wetmur JG, et al. B-Vitamin intake, OneCarbon Metabolism, and survival in a population-based study of women with breast cancer. Cancer Epidemiol Biomarkers Prev. 2008; 17(8):2109-2116.

20. An Y. Association study on polymorphisms of one-carbon pathway with susceptibility of hepatocellular carcinoma [doctoral thesis]. Fudan University; 2008.

21. Gu WJ, Wang F, Tang L, et al. Single-dose etomidate does not increase mortality in patients with sepsis: a systematic review and meta-analysis of randomized controlled trials and observational studies. Chest. 2015; 147(2):335-346.

22. McKeever MP, Weir DG, Molloy A, Scott JM. Betaine-homocysteine methyltransferase: organ distribution in man, pig and rat and subcellular distribution in the rat. Clin Sci. 1991;81(4):551-556. (London).

23. Balinska M, Paszewski A. Betaine-homocysteine methyltransferase in the fungus Aspergillus nidulans. Biochem Biophys Res Commun. 1979; 91(3):1095-1100.
24. Waditee R, Incharoensakdi A. Purification and kinetic properties of betaine-homocysteine methyltransferase from Aphanothece halophytica. Curr Microbiol. 2001;43(2):107-111.

25. Fisher MC, Zeisel SH, Mar MH, Sadler TW. Perturbations in choline metabolism cause neural tube defects in mouse embryos in vitro. FASEB J. 2002;16(6):619-621.

26. Weisberg IS, Park E, Ballman KV, et al. Investigations of a common genetic variant in betaine-homocysteine methyltransferase (BHMT) in coronary artery disease. Atherosclerosis. 2003;167(2):205-214.

27. Hu X, Liu Q, Zhang Z, et al. SHEsisEpi, a GPU-enhanced genome-wide SNP-SNP interaction scanning algorithm, efficiently reveals the risk genetic epistasis in bipolar disorder. Cell Res. 2010;20(7):854-857.

28. Moskvina V, Craddock N, Müller-Myhsok B, et al. An examination of single nucleotide polymorphism selection prioritization strategies for tests of gene-gene interaction. Biol Psychiatry. 2011;70(2):198-203.

29. Lin GT, Tseng HF, Yang CH, et al. Combinational polymorphisms of seven CXCL12-related genes are protective against breast cancer in Taiwan. OMICS. 2009;13(2):165-172.

30. Bei $\mathrm{CH}$, Bai H, Yu HP, et al. Combined effects of six cytokine gene polymorphisms and SNP-SNP interactions on hepatocellular carcinoma risk in southern Guangxi, China. Asian Pac J Cancer Prev. 2014;15(16): 6961-6967.

31. Jin DK, Shido K, Kopp HG, et al. Cytokine-mediated deployment of SDF-1 induces revascularization through recruitment of CXCR4 hemangiocytes. Nat Med. 2006;12(5):557-567.

32. Matsuo K, Wakai K, Hirose K, et al. A gene-gene interaction between ALDH2 Glu487Lys and ADH2 His47Arg polymorphisms regarding the risk of colorectal cancer in Japan. Carcinogenesis. 2006;27(5): 1018-1023.

33. Bendriss-Vermare N, Chaperot L, Peoc'h M, et al. In situ leukemic plasmacytoid dendritic cells pattern of chemokine receptors expression and in vitro migratory response. Leukemia. 2004;18(9):1491-1498.

34. Yen CY, Liu SY, Chen CH, et al. Combinational polymorphisms of four DNA repair genes XRCC1, XRCC2, XRCC3, and XRCC4 and their association with oral cancer in Taiwan. J Oral Pathol Med. 2008; 37(5):271-277.

35. Yin J, Wen J, Hang D, et al. Expression quantitative trait loci for CARD8 contributes to risk of two infection-related cancers-hepatocellular carcinoma and cervical cancer. PLoS One. 2015;10(7):e0132352.

36. Kelemen LE, Terry KL, Goodman MT, et al. Consortium analysis of gene and gene-folate interactions in purine and pyrimidine metabolism pathways with ovarian carcinoma risk. Mol Nutr Food Res. 2014;58(10): 2023-2035.

37. Lin GT, Tseng HF, Yang CH, et al. Combinational polymorphisms of seven CXCL12-related genes are protective against breast cancer in Taiwan. OMICS. 2009;13(2):165-172.

38. Pharoah PD, Dunning AM, Ponder BA, Easton DF. Association studies for finding cancer-susceptibility genetic variants. Nat Rev Cancer. 2004; 4(11):850-860 


\section{Supplementary material}

Table SI The association between BHMT rs3733890 polymorphism and cancer susceptibility in subgroup meta-analysis of cancer types

\begin{tabular}{|c|c|c|c|c|c|c|c|c|}
\hline \multirow[t]{2}{*}{ Polymorphism } & \multirow[t]{2}{*}{ Comparison } & \multirow[t]{2}{*}{ Subgroup } & \multirow[t]{2}{*}{$\mathbf{N}$} & \multicolumn{3}{|c|}{$P$-value } & \multicolumn{2}{|l|}{ Regression model } \\
\hline & & & & PH & $\mathbf{P Z}$ & PE & Random & Fixed \\
\hline \multirow[t]{35}{*}{ rs3733890 } & A vs $G$ & Total & 7 & 0.305 & 0.830 & 0.911 & $0.992(0.915-1.076)$ & $0.992(0.924-1.066)$ \\
\hline & & HNSCC & I & - & 0.544 & - & I.07। (0.859-I.335) & $1.071(0.859-1.335)$ \\
\hline & & $\mathrm{BC}$ & 2 & 0.765 & 0.789 & - & $0.985(0.880-1.102)$ & $0.985(0.880-1.102)$ \\
\hline & & UCC & I & - & 0.017 & - & $0.64 I(0.445-0.923)$ & $0.64 I(0.445-0.923)$ \\
\hline & & oc & I & - & 0.934 & - & $1.015(0.72 \mathrm{I}-\mathrm{I} .427)$ & $1.015(0.72 \mathrm{I}-1.427)$ \\
\hline & & CRA & I & - & 0.346 & - & $1.093(0.909-1.315)$ & $1.093(0.909-1.315)$ \\
\hline & & LC & I & - & 0.780 & - & $0.980(0.852-1.127)$ & $0.980(0.852-1.127)$ \\
\hline & $A A$ vs GG & Total & 7 & 0.203 & 0.878 & 0.706 & I.026 (0.837-I.258) & $1.013(0.863-1.188)$ \\
\hline & & HNSCC & I & - & 0.318 & - & $1.279(0.789-2.073)$ & I.279 (0.789-2.073) \\
\hline & & $\mathrm{BC}$ & 2 & 0.986 & 0.519 & - & $0.921(0.717-1.183)$ & $0.921(0.717-1.183)$ \\
\hline & & UCC & I & - & 0.065 & - & $0.433(0.178-1.054)$ & $0.433(0.178-1.054)$ \\
\hline & & oc & I & - & 0.340 & - & $1.416(0.693-2.894)$ & $1.416(0.693-2.894)$ \\
\hline & & CRA & I & - & 0.104 & - & I.442 (0.928-2.242) & I. $442(0.928-2.242)$ \\
\hline & & LC & I & - & 0.678 & - & $0.935(0.682-1.282)$ & $0.935(0.682-1.282)$ \\
\hline & $A G$ vs $G G$ & Total & 7 & 0.099 & 0.799 & 0.785 & $0.980(0.839-1.145)$ & $0.994(0.894-1.104)$ \\
\hline & & HNSCC & I & - & 0.750 & - & $0.950(0.692-1.304)$ & $0.950(0.692-1.304)$ \\
\hline & & $\mathrm{BC}$ & 2 & 0.554 & 0.658 & - & $1.036(0.886-1.212)$ & $1.036(0.886-1.212)$ \\
\hline & & UCC & I & - & 0.052 & - & $0.614(0.376-1.005)$ & $0.614(0.376-1.005)$ \\
\hline & & OC & I & - & 0.088 & - & $0.647(0.393-1.067)$ & $0.647(0.393-1.067)$ \\
\hline & & CRA & I & - & 0.067 & - & $1.514(0.972-2.360)$ & $1.514(0.972-2.360)$ \\
\hline & & LC & I & - & 0.954 & - & $1.006(0.827-1.224)$ & $1.006(0.827-1.224)$ \\
\hline & $A A+A G$ vs $G G$ & Total & 7 & 0.135 & 0.840 & 0.924 & $0.984(0.855-1.132)$ & $0.990(0.896-1.093)$ \\
\hline & & HNSCC & I & - & 0.947 & - & $1.010(0.749-1.363)$ & $1.010(0.749-1.363)$ \\
\hline & & $\mathrm{BC}$ & 2 & - & 0.880 & - & 1.011 (0.872-I.I73) & $1.011(0.872-1.173)$ \\
\hline & & UCC & I & - & 0.022 & - & $0.579(0.362-0.924)$ & $0.579(0.362-0.924)$ \\
\hline & & OC & I & - & 0.312 & - & $0.788(0.496-|.25|)$ & $0.788(0.496-1.251)$ \\
\hline & & CRA & I & - & 0.071 & - & I.3I3 (0.833-2.070) & $1.313(0.833-2.070)$ \\
\hline & & LC & I & - & 0.929 & - & $0.992(0.823-1.195)$ & $0.992(0.823-1.195)$ \\
\hline & $A A$ vs $A G+G G$ & Total & 7 & 0.334 & 0.895 & 0.572 & $0.995(0.855-1.157)$ & $0.99 \mid(0.865-I .135)$ \\
\hline & & HNSCC & I & - & $0.24 I$ & - & $1.313(0.833-2.070)$ & $1.313(0.833-2.070)$ \\
\hline & & $\mathrm{BC}$ & 2 & 0.867 & 0.416 & - & $0.905(0.7|3-| . \mid 50)$ & $0.906(0.7 \mid 3-1.150)$ \\
\hline & & UCC & I & - & 0.162 & - & $0.54 \mid(0.229-\mid .279)$ & $0.54 \mid(0.229-1.279)$ \\
\hline & & OC & I & - & 0.106 & - & $1.743(0.888-3.420)$ & $1.743(0.888-3.420)$ \\
\hline & & CRA & I & - & 0.853 & - & I.023 (0.803-I.304) & $1.023(0.803-1.304)$ \\
\hline & & LC & I & - & 0.649 & - & $0.933(0.69|-| .259)$ & $0.933(0.691-1.259)$ \\
\hline
\end{tabular}

Note: The values shown in bold indicate that when $P<0.05$, the association between BHMT rs 3733890 polymorphism and cancer risk could be regarded as statistically significant.

Abbreviations: BC, breast cancer; CRA, colorectal adenoma; HNSCC, head and neck squamous cell carcinoma; LC, liver cancer; OC, ovarian cancer; UCC, uterine cervical carcinoma; $\mathrm{PH}, P$-value for heterogeneity test; $P Z, P$-value for $Z$ test (significance test); $P E, P$-value for Egger's test.

\section{Publish your work in this journal}

OncoTargets and Therapy is an international, peer-reviewed, open access journal focusing on the pathological basis of all cancers, potential targets for therapy and treatment protocols employed to improve the management of cancer patients. The journal also focuses on the impact of management programs and new therapeutic agents and protocols on

\section{Dovepress}

patient perspectives such as quality of life, adherence and satisfaction The manuscript management system is completely online and includes a very quick and fair peer-review system, which is all easy to use. Visit http://www.dovepress.com/testimonials.php to read real quotes from published authors. 